\title{
Role of Genetics in Anesthesiology
}

\author{
Ziske Maritska1, Rani Iswara², Ignatius Aldo Winardi², Yuni Dwi Marantika², \\ Irfan Ferdinand Tambunan², Lovina², Mgs. A. Rifqi Murtadho², Sendy Aditya Nugraha², \\ Cendy Legowo², Victor Pulpa Seda², Awang Budi Saksono ${ }^{2}$ \\ ${ }^{1}$ Department of Biology Medicine, Faculty of Medicine, Universitas Sriwijaya, Palembang, Indonesia \\ ${ }^{2}$ Department of Anesthesiology and Reanimation, Faculty of Medicine, Universitas Sriwijaya, Palembang, Indonesia
}

\begin{abstract}
One thing that differentiates one person from another is one's genetic make-up. Genetic plays a role in every branch of medicine, including anesthesiology. An anesthesiologist must be well familiarized with hereditary (genetic) conditions, chromosomal traits, heredity-familial disorders, and even recessive variants because particular conditions might demand a different anesthetic and perioperative pharmacological management. These circumstances may lead to an opening of a rapidly expanding state of pharmacogenetics/genomics and its relevancy in anesthesia nowadays. This narrative review provides insight into the role of genetics in the field of anesthesiology.
\end{abstract}

Keywords: genetics, anesthesiology, pharmacogenetics

\section{Introduction}

Changes in the DNA sequence could alter gene expression, making an individual either suffers from a disease, or prone to a certain condition/disease as a result. The later is called polymorphism. Polymorphisms or the diversity in coding or non-coding region of DNA is $0.1 \%$ in variability. This condition leads to different pharmacokinetics and pharmacodynamics properties in each individual, with consequences like different sensitivity and reactions to anesthesia drugs. This polymorphism differs between one another, where one could contain deletions, and the other could have insertions at multiple or single nucleotides, where all these may affect the clinical pharmacotherapeutics setting. ${ }^{1}$
Polymorphism in certain genes involved in pharmacodynamics and pharmacokinetics can predispose the response of an individual to a drug, including anesthetic drugs. The genetic variation can affect the metabolism or action and binding to the receptor of one another person during the administration of anesthesia drugs. For instance, the diversity of genes in each individual can affect clinical practice, and it is pivotal, especially in anesthesia where polymorphism in certain genes could affect patients' response to anesthesia medication, thus affecting the outcome. ${ }^{1}$ This phenomenon can be called pharmacogenomics, where the body responds to how a person's genetic inheritance is affected. This literature provides a brief review of genetics in anesthesiology from various aspects.

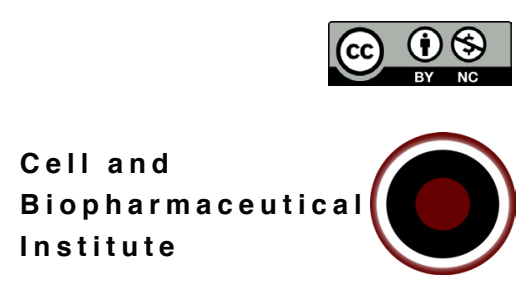

Cell and Institute 


\section{Genetics polymorphisms in pain}

Polymorphism causes differences in nociception function between one another. Although the variability is also influenced by other factors such as sex, psychosocial, environmental, mood, past life experiences, and behavior. Examples of this variability include CYP2D6 gene polymorphism towards codeine and tramadol, CYP2B6 gene polymorphism towards Methadone, and CYP2C9 gene polymorphism towards celecoxib. Based on the studies, there were recommendations made towards the use of these drugs. Take for example, the recommendation made for codein based on the CYP2D6 gene polymorphism where a person can be categorized into ultrarapid, intermediate, and poor metabolizer based on the alleles found. Normal function would have alleles $* 1, * 2, * 27$, and $* 33$ while decreased function would have alleles $* 10, * 17$, *41, and *49. It allows new insight into how an individual's properties of pain medicine in preoperative, intraoperative, and postoperative and chronic pain settings becomes crucial. ${ }^{2,3}$

\section{Genetic and genomic in postoperative pain and choice of treatments}

The increasing prevalence and inadequate efficacy in postoperative pain have become major health issues. During wound healing, many patients feel acute pain after surgery within days to weeks and the rest will develop chronic pain after operative surgery that will last more than three months. There are many factors that develop pain after surgery, such as type of surgery, scared of surgery, anxiety, perioperative nerve injury, pain expectation, obesity, young age, and sex. High pain sensitivity and high risk of postoperative pain had been associated with single nucleotide polymorphism. ${ }^{4}$

The $\mu$-opioid receptor gene (OPRM) are associated with mutation related to analgesic pharmacogenetic. In order to achieve adequate analgesia, most people with homozygous minor allele OPRM1 need 30\% more oxycodone compared with major allele. There are some people with CYP2D6 variation activity that produce morphine efficiently and poorly. ${ }^{4}$ Next Generation Sequencing (NGS) is used to screen for seldom genetic variants that mediate pain. More intensive research in epigenetic changes and postoperative pain is required because it's still poorly understood. Genotype-dependent dosing of analgesics is used to select more effective analgesic treatment. Interindividual variability in drug responses in developing novel analgesic postoperative pain might be facilitated with pharmacogenetics. ${ }^{4}$ Mitochondria are known as agedependent changes in their sensitivity to anesthetics drugs. Aging affects organism response to xenobiotics, mostly in central nervous system. Genome study showed that single nucleotide polymorphism in genes involved in cell water regulation and oxygen detection. ${ }^{5}$

\section{Genetic and awareness with recall during surgery}

Recall during surgery is rare, about $0.2 \%$. Although it is low, it is a disconcerting experience for the patients. The Amnestic effect of general anesthesia occurs at 0.10.3 Minimum Alveolar Concentration (MAC) in $90 \%$ of patients. Recall while under general anesthesia in $10-25 \%$ patients happen in an adequate dose of $>0.5 \mathrm{MAC}$, which shows that anesthetic agents can't alter consciousness and memory formation. Genetic factors may explain resistance to anesthetic drugs in recall while under general anesthesia. Calcium signaling pathways and purinergic pathways have a role in memory formation and arousal. Disruption in CACNA-1H /Cav2. ${ }^{3}$ decreases slow-wave sleep and regulates N-methyl-D-Aspartate receptor function. CACNA-1B makes experimental subjects resistant to propofol and more sensitive to halothane. ${ }^{6}$

\section{Anesthesia consideration in patients with rare genetic diseases}

The focus of perioperative care in patients with coexisting unusual genetic disease begins with thorough evaluation and identification of comorbidities because many congenital anomalies are interconnected.

\section{Airway Abnormalities}

Patients with airway abnormalities have the risk of difficult face mask ventilation and intubation. The etiology of airway abnormalities itself can be caused by varied factors, including bronchopulmonary hypoplasia, altered respiratory drive, gastroesophageal reflux, and also skeletal abnormalities (vertebral and craniofacial anomalies) related to genetics. Thus, in dealing with such cases, the management would be different, where anesthesiologist needs to: (1) evaluate airway for difficult intubation or ventilation before using neuromuscular blocking agents, (2) strictly monitor the patients' airway, (3) prepare the equipment for difficult 
airway, and (4) properly positioning and caring for patients during intubation, such as neck traction, prevent hyperextension and hyperflexion of the cervical spine.

\section{Congenital Heart Diseases}

When handling patients with congenital heart disease, there are at least two things that needs to be done: invasive monitoring during a surgical procedure, and the use of antibiotic prophylaxis to prevent bacterial endocarditis.?

\section{Neuromuscular Disorders}

Anesthesiologists have to carefully select a decent muscle relaxant agents when handling patients with neuromuscular disorders. Succinylcholine can prolong muscle relaxation and cause heart arrhythmias, elevated potassium, respiratory distress, muscle fasciculation, malignant hyperthermia, and joint stiffness. Meanwhile, non-depolarizing muscle relaxants can exaggerate and prolonge response in patients with central nervous system disorders who are hypotonic.?

\section{Alteration of genes expression due to anesthetic agents}

More than 22,000 gene expressions and 129,800 transcripts have been analyzed using oligonucleotide microarrays to study the brain character profile of Sprague Dawley rodents after being given acute and chronic doses of propofol isoflurane and ketamine. In the rodent brain, these three anesthetic agents can cause extensive apoptosis. Isoflurane is an inhaled anesthetic that binds to Gamma-Aminobutyric Acid (GABA), glutamate, and glycine receptors. Rats exposed to isoflurane for two hours can increase the expression of the neocortex and hippocampus. In humans, it is known that anesthesia can affect gene expression from brain memory, including the visual cortex. It is also believed to trigger neuroinflammation.

For example, the elderly can increase the occurrence of precursor lesions that cause Alzheimer's illness and other neurodegenerative diseases. Ketamine is a fast-acting general anesthetic producing profound analgesia, transient respiratory depression, interacts with N-Methyl-D-Aspartate (NMDA). When given in high doses, ketamine can cause nerve cell apoptosis. Propofol is an intravenous anesthetic for induction. Modulate the inhibitory neurotransmitter GABA. Propofol has neuroprotective effects with antioxidant properties, inhibition of GABA transmission, the release of glutamate. ${ }^{8}$
In anesthesia and sedation, GABA-AR Agnois has an important role. Etomidate, propofol, isoflurane, thiopental, methohexital, desflurane, and sevoflurane are examples of GABA-AR agonists used in everyday anesthetic practice. Each drug has its advantages and disadvantages. The most popular anesthetic agent for induction is propofol. Thiopental and etomidate have different geographic variations in their use. The pharmacology of GABA-AR agonists has resulted in new developments based on the chemical configuration that can be well-controlled and well-titrated. This agent has many similarities with other sedative agents used in anesthesia and sedation in the future. ${ }^{9}$

\section{The role of mitochondria in anesthetic agents neurotoxicity}

Pathogenic changes in protein in mitochondrial cells can amplify the chance of disadvantageous effects from general anesthesia has not been earlier investigated. Isoflurane exposure raised mortality in Drosophila (wild-type flies), taking homozygous mutations of the complex one of mitochondrial. Changes in the protein ND-23 subunit of complex one mitochondrial electron carrier string like mammalian species NDUFS- 8 replicate essential features of Leigh syndrome. ${ }^{10}$

Leigh syndrome is a genetic condition where there is changes/mutations in one's mitochondrial DNA. When the mitochondrial DNA is mutated, it will interfere with the energy sources that run cells in an area of the brain that plays a role in motor movements. However, the prevalence of heterozygosity in the global population is substantially higher at 1 to 2:100. This study exposure ND-23 changes flies to equal doses of Isoflurane or Sevoflurane agent in $5 \%, 21 \%$, or $75 \%$. Isoflurane, and sevoflurane agents differentially affected the expression of antioxidant genes in 10 to 13-day old ND-23 flies. The dying of heterozygous ND-23 flies exposed to Isoflurane within $75 \%$ oxygen concentration expands with the period. Disclosure to general anesthesia is widespread but not without dangers. Changes in some anesthetic target proteins enhance the opportunity of toxicity. ${ }^{10}$

\section{Genetics polymorphim in anesthetic management}

For anesthesiologists predicting intubation and preoperative difficulties. Failed tracheal intubation The next step is supraglottic placement. There are four options after insertion 
of a supraglottic airway to wake the patient, tracheal intubation via a supraglottic airway continued without tracheal intubation or tracheostomy/cricothyroidotomy. ${ }^{11}$ Management of bleeding according to the American Society of Anesthesiologists $7.0 \mathrm{~g}$. Several articles on the anesthetic management of children with FTHS are challenging because major scoliosis surgery is a very important preoperative preparation to control difficult intubation and bleeding. It is recommended before the relevant surgery is consulted and considered. ${ }^{11}$

\section{Genetic polymorphisms in Frank-Ter Haar syndrome}

Frank-Ter Haar syndrome is rare autosomal recessive characterized by skeletal, developmental delay, cardiovascular, ocular abnormalities, and facial features such as a very large cornea, broad fontanel, prominent forehead, eye spacing) on chromosome $5 \mathrm{q} 35.1$ by a mutation in the SH3PXD2B, the gene is Frank-Ter Haar syndrome. ${ }^{11}$

Frank and Ter Haar in 1973 explained that congenital syndromes affecting the skeletal system such as metacarpal bone deformities, scoliosis kyphosis, osteolysis, and vertebral beaks as well as in the eye such as macro corner with or without glaucoma and facial features are associated with brachydactyly, hypertelorism. ${ }^{11}$

\section{Genetic polymorphisms in factor $\mathrm{X}$ deficiency}

Intrinsic factor $\mathrm{X}$ deficiency is a rare condition clotting disorder with an occurrence number of 1:1000000 in the population. Factor $\mathrm{X}$ serum protease synthesized in the liver is vitamin $\mathrm{K}$ dependent, and deficiency in factor $\mathrm{X}$ is an uncommon acquired congenital coagulopathy. It is the first coagulation factor in the common pathway; when stimulated, factor $\mathrm{X}$ catalyzes the transformation of prothrombin into thrombin. Fertility in women with factor X deficiency d is usually correlated with unfavorable outcomes, including spontaneous abortion, premature labor, and bleeding during pregnancy and peripartum time. The American Society of Anesthesiologists (ASA) Task Force on perioperative blood Management declares that FFP indicates or improves excessive microvascular bleeding conditions. ${ }^{12}$

\section{Genetics in heredity arrhythmia}

Tendency to sudden cardiac death (SCD) and potential duration due to the influence of cardiac ion channels from the congenital abnormality is congenital long QT syndrome. There are two classic hereditary modifications, Romano Ward syndrome, which is variable penetration and autosomal dominant, also Jervell and Lange Nielson syndrome lives autosomal recessive, much less common. In unit point from origin to adulthood similar symptoms, often into puberty and teens. Illnesses, beats, or syncope occasioned by torsade de pointes, some polymorphic form of ventricular tachycardia, are the diagnosis is selflimiting usually lasts less than one minute but may grow to ventricular fibrillation.13 Other form of inherited arrhytmias are: (1) $\mathrm{Na}^{+}$channelopathies, (2) arrhytmias due to $\mathrm{K}^{+}$ channel mutations, and (3) arrhytmias due to other inherited arrhytmogenic mechanism. ${ }^{14}$ These conditions are caused by different mutations, for example, $\mathrm{Na}^{+}$channelopathies are caused by mutations in SCN5A, with variable expressivity and penetrance, meaning its clinical manifestation may differ from one person to another. ${ }^{14}$

Diagnostic with a pathogenic mutation in the setting of LQTS. Differential diagnosis, which can prolong QTc is hypokalemia, hypomagnesemia, hypocalcemia, and hypothyroidism-left ventricular dilatation as a cause of obtained long QT syndrome. Decreased cardiac potassium channel activity or increased sodium or calcium channel activity results in the potential for heterogeneity and elongation induced by LQTS mutation. Management strategies age 40 have an annual occurrence of cardiac arrest or sudden death, and QTc shortens, males usually at greater risk unto puberty. The main treatment is $\beta$-blocker, specifically propranolol or nadolol, designated at the highest dose. During patients with LQT2, electrolyte imbalances should be avoided because they are susceptible to hypokalemia and medications that can increase the QT interval. $^{13}$

\section{Anesthetic involvement of the alteration superintendence of mucopolysaccharidosis (MPS) patient}

MPS is a group of inborn error metabolism condition where the body can not breakdown mucopolysaccharides properly. MPS is a long chain made of sugar molecules that are ubiquitous in human's body. Since the body can not break it down, the molecules will be buildup in cells, that eventually lead to various clinical manifestations. There are several types of muccopolysaccharidosis (muccopolysaccharidosis type I, type II, type III, type IV, type V, type VI, type VII, 
and type IX). However, a lot of its cases are passed on in an autosomal recessive patern, with only type II passed on in an X-linked pattern. ${ }^{15}$

The mucopolysaccharidoses are inbred metabolic disturbances noted to provide clinical matter, mainly corresponded to airway, cardiac and skeletal anomaly, in children in the course of anesthesia. The alteration clinical superintendence of the mucopolysaccharidosis can be explained in three stages. An opening stage of hoarding and deployment of learning about superintendence of this rare illness with an accretion confession that not treated MPS type I and with the critical structure of other types like Maroteaux syndrome were connected with acute complications under anesthesia. The second stage followed this mirroring the valuable outcome treatment like hemopoietic stem cell transplantation therapeutics. ${ }^{16}$

\section{Electrocardiography impact of propofol against etomidate in Brugada Syndrome patient}

Brugada syndrome is a genetic condition where a person who suffered from it could experience ventricular tachyarrhytmias that can lead to cardiac arrest or sudden cardiac death. As a genetic condition, Brugada syndrome is passed down in an autosomal dominant manner, meaning it only takes one affected parent to have a 50:50 chance of having an affected child. Up to this date, this condition is known to be caused by mutations in eight genes (SCN5A, GPDIL, CACNA1C, CACNB2, SCN1B, KCNE3, SCN3B, and $\mathrm{HCN} 4)$. The diagnosis of Brugada syndrome can only be established by investigating the genetic cause. ${ }^{17}$

There was no meaningful diversity in electrocardiographic changes in Brugada syndrome patients when propofol against etomidate was administered to induce anesthesia. This research did not find out electrocardiographic changes concerned to propofol used as a treatment for anesthesia keeping, so future research would be guaranteed before inference about the safety protocol treatment in Brugada syndrome patients can be determined. ${ }^{18}$

\section{Children with medium-chain acetyl-CoA dehydrogenase deficiency}

Medium-Chain-Acyl-Coenzyme A Dehydrogenase (MCAD) is an enzyme playing a role in mitochondrial fatty acid ß-oxidation. Lack of this enzyme could lead to hypoketotic hypoglycemia that manifests in lethargy, seizures, and coma. MCAD deficiency is one of the most commonly found inborn errors of metabolism in children. In well developed countries, most children are screened and diagnosed through newborn screening. MCAD deficiency is caused by mutations in ACADM gene that can be identified through molecular testing. It is inherited in an autosomal recessive manner where carrier parents are at risk for having an affected child as big as $25 \% .{ }^{19}$

In healthy children taking glucose supplementation, perioperative metabolic decompensation is rare. Surgery and glucose supplementation can cause blood sugar levels to rise. Found no significant complications with bolus administration of propofol and the volatile anesthetic agent. Atracurium, a muscle relaxant, if used for a long time, can cause delayed offset in patients. ${ }^{15}$ As recommended, administration of glucose supplements, frequent perioperative blood sugar monitoring, and neuromuscular blockade are recommendations to this group of patients. No specific approach to anesthesia would be recommended. ${ }^{20}$

\section{Genetics in Donohue syndrome}

Donohue syndrome is an inherited disease that causes defects in insulin receptor function, an autosomal recessive disorder that affects the ability of insulin to bind to receptors. It is caused by mutations in INSR gene that can be passed on in an autosomal recessive pattern, meaning parents of an affected individual needs to carry one copy of the mutated gene. There is little relevant literature to date. about anesthesia considerations in Donohue syndrome, and anesthetic management in Donohue syndrome. One of the case series reporting five children with Donohue syndrome showed that the patients are at risk of general anesthesia. The risks mainly related to restrictive lung disease and abdominal distention that could be getting worse due to bag valve mask ventilation and limited respiratory reserve. $^{21}$

A spontaneous breathing technique is highly recommended in the induction phase. Bag valve ventilation is usually used in patients with constant gastric aspiration and defying intubation. There is failure to thrive in patients with this syndrome and low birth weight, causing various risks in anesthesia such as difficult intravenous access, hypoglycemia, and hypothermia. The effect of anesthesia on children suffering from this syndrome can cause cardiomyopathy, causing cardiovascular collapse. ${ }^{21}$ 
The presence of abdominal distension and a small thoracic diameter causes decreased chest compliance and problems with bag-valve ventilation. In difficult mask ventilation, we must prepare additional airway devices such as a laryngeal mask airway that can be used if there is an obstruction in the airway. If difficulty in airway planning, we can ask for help from a more expert senior, use an indirect laryngoscope, and involve the ENT team if needed. ${ }^{21}$

\section{Anesthetic consideration in Congenital Insensitivity to Pain with Anhidrosis (CIPA) and skeletal dysplasia}

\section{Congenital Insensitivity to Pain with Anhidrosis (CIPA)}

CIPA is an autosomal recessive disorder characterized by an elevated temperature, no sweating, and an absence of sensitivity to pain sensations. It is a disorder of functional mutations in TKRA acting on the neurotrophic receptor tyrosine kinase type 1 and the receptor on the nNerve Growth Factor (NGF), causes the NGF to be unable to bind to Neurotrophic Receptor Tyrosine Kinase 1 (NTRK1) resulting in decreased TKRA signaling and death in specific nerves. ${ }^{22}$ This process can occur at the stage of embryonic formation, causing insensitivity to pain and disorders of the autonomic nervous system. There are case reports in China showing that often, there is an increase in temperature during surgery, an increase in blood pressure, and bradycardia due to the influence of autonomic dysfunction; this can last for 6 hours after surgery. So that in some cases, patients with this condition do not require opiates during surgery because they already have congenital analgesia. ${ }^{23}$

\section{Skeletal Dysplasia}

Skeletal dysplasia is a disorder that occurs in the development and growth of the human skeleton that is heterogeneous and common. It is a group of more than 400 inherited bone disorders, with many genes involved as well. Skeletal dysplasias can be inherited in various manner, namely: autosomal recessive, autosomal dominat, $\mathrm{X}$-linked recessive, $\mathrm{X}$-linked dominant, and Y-linked. This disorder is a big problem that can occur during anesthesia management because it involves several abnormalities of the airway, either morphologically or functionally, and limitations in abnormal mobility of the cervical spine. There are a few things to consider when the patient: (1) has small chest space and narrow airways, (2) has inappropriate craniofacial growth that can lead to jaw malformations and restriction of mobilization during intubation, (3) is overweight. Genetic mutations can cause characteristic deformities of bone, non-skeletal organ systems. Collagen II mutations can cause tracheomalacia, laryngomalacia, and eyeball fragility, increasing the risk of anesthesia. To screen for skeletal dysplasia before undergoing anesthesia should be screening neurological examination, radiographic examination. Patients with skeletal dysplasia tend to have frequent hypoxemia, thus sedation for preoperative is recommended. Furthermore, for airway management, a use of video laryngoscope for intubation is also recommended. One thing to notice is patients with skeletal dysplasia are not recommended for surgery under the management of epidural anesthesia. ${ }^{24}$

\section{Genetics in malignant hyperthermia}

Malignant hyperthermia is a dangerous reaction of high body temperature reaction when doing general anesthesia. Some people genetically prone to this disease have an increased risk of malignant hyperthermia if given anesthetics gas such as suxamethonium. It can be called a malignant hypermetabolic syndrome. There are no unique symptoms of malignant hyperthermia, and it can kill the bearer if it is not treated in the early stages. The treatment needs to be aggressive and given as soon as possible. The Association of anesthetists already made instructions in the anesthetics room to guide if malignant hyperthermia occurs. The last principle was in 2011 and has been updated. In this instruction, we will give information and recommendations for this situation. This instruction has complete information for anesthetists when dealing with this disease. After the crisis, the information about care and monitoring need tools and how to resurrect patients in the operating room and ICU, communication, teamwork, and instruction on handling patient family. ${ }^{25}$

With the inventions of genes related to malignant hyperthermia, including ryanodine receptor 1 (RyR1), CACNA1A, and STAC3, and improvement in genetics screening tools, including new sequencing, our knowledge of the genetics of malignant hyperthermia has increased. The new sequencing method has made genetic testing possible for malignant hyperthermia screening for first-line diagnostic test for patients with malignant hyperthermia reaction under anesthesia. With the low price of the sequencing, the chance of rare case of RYR1 and CACNA1S and other genetic disorders can be found more easily. ${ }^{26}$ 


\section{Current Genetic Testing Available}

Genetic testing and genomic testing have been growing rapidly since the completion of human genome project in 2003. Nevertheless, the availability and accessibility of said testing are not yet widespread. Genetic and genomic tests are applied based on what is being analyzed; single genes, multiple genes, exomes, or an entire genome. Common genetic testing involved single-gene test, especially for single-gene disease that are inherited in a Mendelian inheritance pattern. For a single-gene test, a simple Polymerase Chain Reaction (PCR) can be applied. However, in diseases with polygenes involvement, a panel tests of multiple genes using microarray is preferred. When examining the whole exome, a whole exome sequencing is preferrable, where the test would evaluate the coding regions only. Meanwhile, if the genetic cause can not be determined, a whole genome analysis can be conducted to investigate the whole genome, both coding and non coding regions. ${ }^{27}$

\section{Conclusion}

Although rarely discussed, genetics affect anesthesiology in many ways. Certain genetic variants can alter one's response to particular medication, thus affecting the patients' outcome. Identifying genetic polymorphism can be made possible through genetic and genomic testing. However, it is recommended for those working on the anesthesiology field to be aware of such conditions that might demand a different anesthetic and perioperative pharmacological management.

\section{References}

1. Panditrao MM, Panditrao MM. Editorial: anaesthesia and genetics: still, the uncharted territories!! J Anesth Crit Care Open Access. 2016; 4(1): 00123. doi: 10.15406/jaccoa.2016.04.00123.

2. Packiasabapathy S, Horn N, Sadhasivam S. Genetics of perioperative pain management. Curr Opin Anaesthesiol. 2018; 31(6): 749-55.

3. Pratt VM, Scott SA, Pirmohamed M, editors. Codeine therapy and CYP2D6 genotype. In: Medical Genetics Summaries [Internet]. Bethesda: National Center for Biotechnology Information (US); 2012. Available from: https://www.ncbi.nlm.nih.gov/books/ NBK100662/.

4. PaladaV, Kaunisto Ma, Kalso E. Genetics and genomics in postoperative pain and analgesia. Curr Opin Anaesthesiol. 2018 31(5): 569-74

5. Schiffman HJ, Olufs ZPG, Lasarev MR, Wassarman DA, Perouansky $M$. Ageing and genetic background influence anaesthetic effects in a D. melanogaster model of blunt trauma with brain injury†. Br J Anaesth. 2020; 125(1):77-86.

6. Sleigh JW, Leslie K, Davidson AJ, Amor DJ, Diakumis P, Lukic $\mathrm{V}$, et al. Genetic analysis of patients who experienced awareness with recall while under general anesthesia. Anesthesiology. 2019; 131(5): 974-82.

7. Oliveira, Degrandi CR. Anesthesia in patients with unusual genetic diseases. Anaesth Pain Intensive Care. 2019; 23(1): 5-8.

8. Upton DH, Popovic K, Fulton R, Kassiou M. Anaesthetic-dependent changes in gene expression following acute and chronic exposure in the rodent brain. Sci Rep. 2020; 10(1): 9366. doi: 10.1038/s41598020-66122-6.

9. Brohan J, Goudra BG. The role of GABA receptor agonists in anesthesia and sedation. CNS Drugs. 2017; 31(10): 845-56.

10. Olufs ZPG, Ganetzky B, Wassarman DA, Perouansky M. Mitochondrial complex I mutations predispose drosophila to isoflurane neurotoxicity. Anesthesiology. 2020; 133(4): 839-51. doi: 10.1097/ALN.0000000000003486.

11. Basaran I, Gozubuyuk E, Canbolat N, Edipoglu IS, Buget MI. Anesthetic management of scoliosis operation in a pediatric patient with Frank-ter Haar syndrome: a case report. Braz J Anesthesiol. 2021; 71(2): 181-3.

12. Krkovic M, Koosova Gajic A, Tarčuković J, Sotosek V, Stimac $\mathrm{T}$, Balen $\mathrm{S}$, et al. Factor $\mathrm{X}$ deficiency management for elective cesarean delivery in a pregnant patient. Am J Case Rep. 2020; 21: e920685. doi: 10.12659/AJCR.920685

13. Levy D, Bigham C, Tomlinson D. Anaesthesia for patients with hereditary arrhythmias; part 2: congenital long QT syndrome and arrhythmogenic right ventricular cardiomyopathy. BJA Educ. 2018; 18(8): 246-53. doi: 10.1016/j.bjae.2018.04.005.

14. Lehnart SE, Ackerman MJ, Benson DW Jr, Brugada R, Clancy CE, Donahue JK, et al. Inherited arrhythmias: a National Heart, Lung, and Blood Institute and Office of Rare Diseases workshop consensus report about the diagnosis, phenotyping, molecular mechanisms, and therapeutic approaches for primary cardiomyopathies of gene mutations affecting ion channel function. Circulation. 2007; 116(20): 2325-45.

15. Hirst L, Mubeen S, Abou-Ameira G, Chakrapani A. Mucopolysaccharidosis (MPS): Review of the literature and case series of five pediatric dental patients. Clin Case Rep. 2021; 9(3): 1704-10.

16. Hack HA, Walker R, Gardiner P. Anaesthetic implications of the changing management of patients with mucopolysaccharidosis. Anaesth Intensive Care. 2016; 44: 660-8

17. Sheikh AS, Ranjan K. Brugada syndrome: a review of the literature. Clin Med. 2014; 14(5): 482-9.

18. Flamée P, Varnavas V, Dewals W, Carvalho H, Cools W, Bhutia JT, et al. Electrocardiographic effects of propofol versus etomidate in patients with Brugada syndrome. Anesthesiology. 2020; 132: 44051.

19. Merritt JL 2nd, Chang IJ. Medium-chain acyl-coenzyme A dehydrogenase deficiency. In: Adam MP, Ardinger HH, Pagon RA, editors. GeneReviews ${ }^{\circledR}$ [Internet]. Seattle: University of Washington, Seattle; 1993-2022. Available from: https://www.ncbi. nlm.nih.gov/books/NBK1424/.

20. Allen C, Perkins R, Schwahn B. A retrospective review of anesthesia and perioperative care in children with medium-chain acyl-CoA dehydrogenase deficiency. Paediatr Anaesth. 2017; 27(1): 60-5.

21. Kirkwood A, Stuart G, Harding L. Donohue syndrome: A review of literature, case series, and anesthetic considerations. Paediatric Anaesth. 2018; 28(1): 23-7.

22. Mughal SM, Farhat A. Case study of a rare genetic disorder: congenital insensitivity to pain with anhidrosis. Cureus. 2021; 13(1): e12984. doi: 10.7759 /cureus. 12984 
23. Wang C, Zhang X, Guo S, Sun J, Li N. Anesthetic management during adenotonsillectomy for twins with congenital insensitivity to pain with anhidrosis: two case reports. J Med Case Reports. 2017; 11: 247. doi: 10.1186/s13256-017-1406-0.

24. White KK, Bompadre V, Goldberg MJ, Bober MB, Cho TJ, HooverFong JE, et al. Best practices in peri-operative management of patients with skeletal dysplasias. Am J Med Genet A. 2017; 173(10): 2584-95.
25. Hopkins PM, Girard T, Dalay S, Jenkins B, Thacker A, Patteril M, et al. Malignant hyperthermia 2020: Guideline from the Association of Anaesthetists. Anaesthesia. 2021; 76(5): 655-64.

26. Riazi S, Kraeva N, Hopkins PM. Malignant hyperthermia in the postgenomics era: new perspectives on an old concept. Anesthesiology. 2018; 128(1): 168-80.

27. Phillips KA, Deverka PA, Hooker GW, Douglas MP. Genetic test availability and spending: Where are we now? Where are we going? Health Affairs. 2018; 37(5): 710-6. 